\title{
The Potential Spread of Mycobacterium tuberculosis into the Environment in the Creation of Spondylitis Tuberculosis Rabbit
}

\author{
Ahmad Jabir Rahyussalim, ${ }^{1}$ Tri Kurniawati, ${ }^{2}$ Andriansjah Rukmana, ${ }^{3}$ and Arni Diana Fitri ${ }^{4}$ \\ ${ }^{1}$ Orthopaedic and Traumatology Department, Faculty of Medicine, Cipto Mangunkusumo Hospital, University of Indonesia, \\ Jakarta 10430, Indonesia \\ ${ }^{2}$ Integrated Service Unit of Stem Cell Medical Technology, Cipto Mangunkusumo Hospital, Jakarta 10430, Indonesia \\ ${ }^{3}$ Microbiology Department, Faculty of Medicine, University of Indonesia, Jakarta 10330, Indonesia \\ ${ }^{4}$ Veterinary Teaching Hospital, Bogor Agricultural Institute, Bogor, West Java 16680, Indonesia
}

Correspondence should be addressed to Ahmad Jabir Rahyussalim; rahyussalim71@ui.ac.id

Received 3 July 2015; Revised 17 August 2015; Accepted 31 August 2015

Academic Editor: Alistair Bishop

Copyright (C) 2015 Ahmad Jabir Rahyussalim et al. This is an open access article distributed under the Creative Commons Attribution License, which permits unrestricted use, distribution, and reproduction in any medium, provided the original work is properly cited.

\begin{abstract}
Direct Mycobacterium tuberculosis inoculation on rabbit vertebral body was used in rabbit spinal infection study. The potential spread of Mycobacterium tuberculosis into the environment will be observed in order to create the conditions fulfilling biosafety aspects. Two groups of six New Zealand rabbits were treatment group $(n=4)$ and control group $(n=2)$. The treatment group had injection of $0.1 \mathrm{~mL}\left(10^{7} \mathrm{cfu} / \mathrm{mL}\right)$ suspension of Mycobacterium tuberculosis into the vertebral body T12. They were incubated for 2 to 14 weeks. One rabbit per period of 2, 4, 6, and 14 weeks was euthanized to collect feces, urine, saliva, and tissue lesions. The control group had only feces, urine, and saliva to detect bacteria using AFB staining, culture, and PCR. Both two groups were kept in individual cages. They were put together in a large cage for 3 hours every day to interact with each other. AFB staining, culture, and radiological examination showed negative result, but in one rabbit, histopathological examination showed positive result and PCR examination in another rabbit of the treatment group. Spreading score was 1.05\% and infected score was 0 (null). The procedure did not reveal the potential spread of Mycobacterium tuberculosis into the environment.
\end{abstract}

\section{Introduction}

Spondylitis tuberculosis is an infection disease of the spine caused by Mycobacterium tuberculosis. Spondylitis tuberculosis results in corpus defects that lead to spinal instability and disruption of surrounding structures. The healing of bacterial infections in cases of spondylitis tuberculosis is influenced by the severity of the corpus defects and the bacterial infection in the spine $[1,2]$. All this time, the treatment efforts of spondylitis tuberculosis with corpus defects have been done through the operative approach and the addition of graft (scaffold) [2], but the treatment, in some cases, gives unsatisfactory results because of the failure to achieve fusion due to the absence of new bone formation; therefore, new methods need to be developed to achieve more satisfactory treatment.
Rabbits are models that are often used as experimental animals in various studies in terms of the relative merits and its historical context with human diseases. A study of Manabe et al. [3], Converse et al. [4], and Tsenova et al. [5] showed that white rabbits of New Zealand strain can be infected by Mycobacterium tuberculosis using aerosol infection system after being exposed for 6-33 weeks. On the other hand, as animal models of spondylitis tuberculosis, rabbits are chosen because they have the size of the spine that is not too small so that it will facilitate the induction of infection or other interventions [6].

In the experiment of creation of animal models infected by the Mycobacterium tuberculosis in the lungs (lung tuberculosis), a closed aerosol pump system is used which requires all experimental procedures to be performed in the facilities of Animal Bio Safety Level 3 (ABSL3) with the aim of 
fulfilling aspects of biosafety and biosecurity [5, 7]. While for the study of the same bacterial infection to the corpus of the spine with direct inoculation method (nonaerosol), it is debatable whether the experimental procedure shall also be conducted in facilities of ABSL3. In certain circumstances where the facilities of ABSL3 are limited or not possible to use, the opportunity to perform of the primary infection at facilities other than ABSL3 should be learned. In this study, the potential spread of Mycobacterium tuberculosis into the environment from a rabbit model of spondylitis tuberculosis was observed, in order to create the conditions and methods fulfilling aspects of biosafety and biosecurity.

\section{Material and Methods}

The study was an interventional study on rabbits that have met the ethical review and have obtained the approval from Animal Care and Use Committee (ACUC) of PT Bimana Indomedical and Animal Hospital of Bogor Agricultural Institute. Most of this research used animal facilities at RSH IPB, PT Bimana Indomedical and Clinical Microbiology Laboratory of Faculty of Medicine University of Indonesia (FMUI).

The selection of rabbit samples was based on the rabbit's body weight, bone maturity, sex, and clinical, radiological, and laboratory examination where the inclusion criteria were white rabbits of New Zealand strains weighing 2500-3500 grams, which were healthy and skeletally mature, whereas the exclusion criteria were rabbits with congenital spine abnormalities and/or abnormalities in the spine caused by infections, trauma, neoplasm, and so forth.

Before being used in this study, Mycobacterium tuberculosis was activated by making a suspension in Middlebrook's liquid medium and being incubated in a shaker with a speed of $150 \mathrm{rpm}$, temperature of $37^{\circ} \mathrm{C}$ for 18 hours. The bacterial suspension was then diluted using sterile physiological water to obtain a number of bacteria of $10^{7} \mathrm{cfu} / \mathrm{mL}$ (colony forming units/milliliter) or equivalent to $0.1 \mathrm{~A}$ in the measurement of Optical Density (OD) at wavelength of $600 \mathrm{~nm}$. The treatment for the bacteria followed standard operating procedures applied in Clinical Microbiology Laboratory of FMUI.

Six rabbits were divided into two groups: treatment group $(n=4)$ and control group $(n=2)$. The induction procedure of bacterial infection of Mycobacterium tuberculosis was performed to four rabbits of the treatment group, while to two rabbits of the control group, the induction procedure of infection was not performed.

Four rabbits of treatment group (G3, G4, G5, and G6) were shaved on their back as high as T12 to L1 and were then given anesthetic mixture of ketamine $\mathrm{HCl}(44 \mathrm{mg} / \mathrm{kg})$ and xylazine $(5 \mathrm{mg} / \mathrm{kg})$ intramuscularly. After the anesthetic worked, the rabbits were faced to the side (the left side of the back facing the surgery operator), and then antiseptic and aseptic procedures were performed using 70\% alcohol and betadine on the shaved back of the rabbits, and then they were given sterile cloth. Identification of thoracal 12 was performed by touching the 12th rib and being traced to the transverses process, transverse incision was then performed starting from the spinous process of $3-5 \mathrm{~cm}$ width directing to the left lateral penetrating cutis and subcutis. Paraspinal muscles were separated reaching the 12 th rib, the transverses process, and lamina thoracal 12. Reidentification was performed to determine the position of thoracal 12 before a hole was made using a drill at the midpoint of the corpus thoracal 12 ( $+5 \mathrm{~mm}$ from the transverses process) of $6-10 \mathrm{~mm}$ depth using the drill bit of $1.5 \mathrm{~mm}$. One-tenth $\mathrm{mL}$ suspension of Mycobacterium tuberculosis $\left(10^{7} \mathrm{cfu} / \mathrm{mL} \sim 0.1\right.$ absorbance) was injected aseptically into the hole made in the corpus, then the hole was covered by subcutis fat using a root dissector, surgical wound was covered layer by layer, and skin was sewn one by one using vycril 3.0 thread. Surgical wound was then covered by bandages and the rabbits were returned to the cage and were given ketoprofen $(3 \mathrm{mg} / \mathrm{kg})$ intramuscularly every 12 hours for 3 days. Four rabbits were then maintained/incubated in individual cages for 2, 6, 10, and 14 weeks. While the two rabbits of control group (G1 and G2) were also kept in individual cages and were interacted with the rabbits of treatment group for 3 hours per day.

Clinical examinations were performed, namely, observations of daily activities, appetite, paralysis, signs of infection (the presence of sinus, abscess), and measurement of body weight every three days on all treatment and control rabbits. Sampling of feces, urine, and saliva was done for Acid Fast Bacilli (AFB) staining which refers to grading of AFB smears as per WHO and IUATLD recommendation, examination of Polymerase Chain Reaction (PCR), and culture at the end of weeks 2, 6, 10, and 14 after infection induction procedures for all rabbits (control and treatment). At the end of weeks $2,6,10$, and 14, radiological examination was performed to one rabbit from treatment group per each period; the rabbit was then euthanized using lethal injection at a dose of $150 \mathrm{mg} / \mathrm{kg}$ intravenously (IV). Necropsy was then performed to the rabbit and sample of lesion tissue at thoracal 12 was taken for AFB staining, and examinations of PCR, culture, and histopathology.

PCR amplification for IS6110 gene was performed by set of primers TB1,5-CTC GCGAGCGTAGGCGTCGG-3' ${ }^{\prime}$ and TB2,5' -CTCGTCCAGCGCCGCTTCGG-3', which amplify a fragment of 130-base pair (bp) of the target gene. DNA amplification was performed in a Biorad thermocycler, in a final volume of $20 \mu \mathrm{L}$ containing 10x PCR buffer, $25 \mathrm{mM} \mathrm{Mg}$ $\mathrm{Cl}_{2}, 10 \mathrm{mM}$ dNTPs, $10 \mu \mathrm{M}$ of each primer, $0.12 \mu \mathrm{L}$ HotStar DNA polymerase, and $4 \mu \mathrm{L}$ of template DNA. The amplification program was consisted of initial denaturation at $95^{\circ} \mathrm{C}$ for $5 \mathrm{~min}$, followed by 40 cycles of denaturation at $94^{\circ} \mathrm{C}$ for $30 \mathrm{~s}$, annealing at $60^{\circ} \mathrm{C}$ for $30 \mathrm{~s}$, extension at $72^{\circ} \mathrm{C}$ for $30 \mathrm{~s}$, and a final extension at $72^{\circ} \mathrm{C}$ for $5 \mathrm{~min}$.

PCR examination was performed on liquid (urine, saliva) and solid (stools, lesion tissues) specimens. For urine, the procedure was started by centrifugation at $12,000 \mathrm{rpm}$ for 5 minutes. The supernatant was discarded. DNA contained in the pellets was isolated according to the manufacturer's instructions (QIAmp DNA kit, Qiagen). For Saliva, because the numbers of saliva was a bit, then the centrifugation steps were eliminated. So that the isolation of DNA in saliva was done directly on the samples in accordance with the manufacturer's instructions (QIAmp DNA kit, Qiagen). For 
TABle 1: Potential spread/infected score.

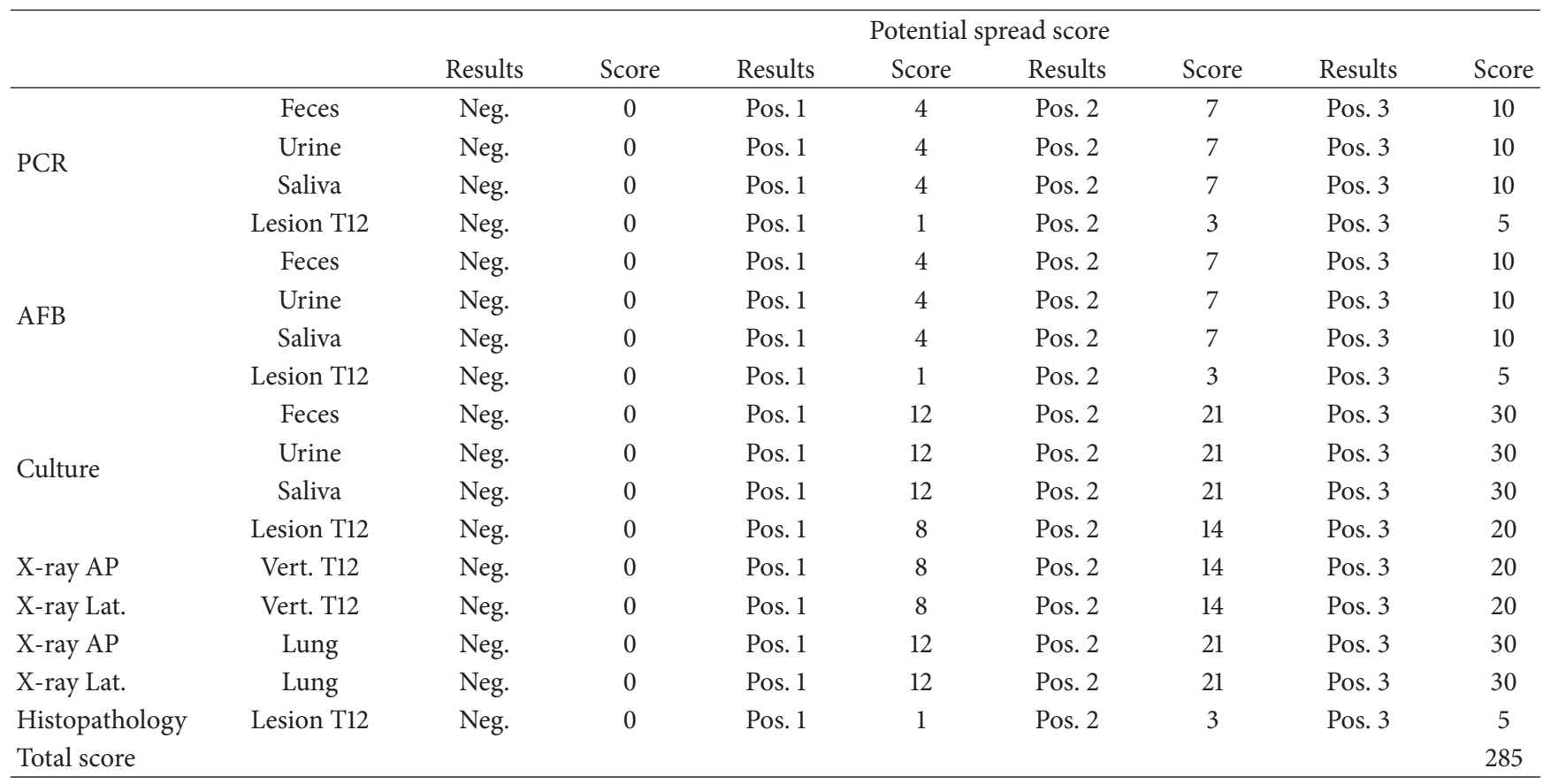

Score from 0 to 9 is not infected; score from 10 to 39 is mildly infected; score from 40 to 69 is infected; score from 70 to 89 is heavily infected; score from 90 to 100 is very heavily infected.

Score from 0 to 9 is not potential; score from 10 to 39 is low potential; score from 40 to 69 is medium potential; score from 70 to 89 is high potential; score from 90 to 100 is very high potential.

Formula of spread/infected score: Potential spread/Infected $=($ Mean score/Total score $) \times 100 \%$.

solid samples such as tissue lesions and feces, the stool samples were weighed to obtain $200 \mathrm{mg}$. Isolation of DNA was then performed according to the manufacturer's instructions (DNeasy Blood And Tissue Kit, Qiagen).

The results of the examination on feces, urine, and saliva and tissue lesion samples were calculated using scoring systems, which were potential spread scoring and infected scoring. Potential spread scoring was calculated from the examination results obtained from the treatment rabbits, while infected scoring was calculated from the examination results obtained from control rabbits. The description on this scoring system can be seen in Table 1 .

There are 8 modalities examination in this study, namely, PCR, AFB, culture, AP thoracolumbar X-ray, lateral thoracolumbar X-ray, AP lung X-ray, lateral lung X-ray, and histopathology examination. Authors made a rule that any test results should be reported as fourth grade which were divided into negative, positive 1, positive 2, and positive 3 . Each grade is given particular weight assigned by authors (Table 1).

Potential and infected scores were developed based on the premise that more specimens were examined (feces, urine, saliva, and lesions) and examination modalities (AFB, PCR, culture, imaging, and histopathology) used the more accurate the prediction results of potential spread score and infected score. We did a weighting to each modality examination and the type specimens to be examined (Table 2). It was based on the experience of authors and literature reviews of all diagnostic methods used in this study.
We provide a high weight for culture examination of urine and saliva and X-ray examination of lung (score 30) with the consideration that the potency for disease transmission from urine and saliva specimens larger than the other, while histological examination and PCR examination of vertebral lesions got the lowest weight (score 5).

\section{Results}

The results of AFB staining, PCR, and culture examination on feces, urine, and saliva samples were obtained. All these examinations showed negative. In addition, the data of AFB staining, PCR, culture (Table 3), and histopathology (Figure 1) examination of lesion tissue specimens and the results of X-ray images (Figure 2) of the rabbits' spine after the incubation period specified were also obtained. Since treatment rabbit 3 (G3) which should be euthanized at week 2 was dead in week 1 , then the data of examination results were adjusted to the data of weeks $1,6,10$, and 14 . Rabbit G3 was dead in week 1 due to surgical trauma. It suffered no appetite after inoculation, its activity was, reduced and then it lost weight until death. PCR examination of lesion tissue from Rabbit G3 showed positive 3 and histopathological examination of lesion tissue from rabbit G4 showed positive 2, while others showed negative. Negative results indicated that there was no Mycobacterium tuberculosis found in examination through the method used in this study, whereas positive results indicated the presence of Mycobacterium tuberculosis on the examination through the method used. 
TABLE 2: Equivalency of examination results with score value.

\begin{tabular}{|c|c|c|c|c|c|c|}
\hline \multirow{2}{*}{ Examination } & & \multirow{2}{*}{ Sample } & \multicolumn{4}{|c|}{ Examination results } \\
\hline & & & Negative $1(-)$ & Positive $1(+1)$ & Positive $2(+2)$ & Positive $3(+3)$ \\
\hline \multirow{17}{*}{ Scoring } & \multirow{4}{*}{ PCR TB } & Feces & 0 & 4 & 7 & 10 \\
\hline & & Urine & 0 & 4 & 7 & 10 \\
\hline & & Saliva & 0 & 4 & 7 & 10 \\
\hline & & Lesion T12 & 0 & 1 & 3 & 5 \\
\hline & \multirow{4}{*}{$\mathrm{AFB}$} & Feces & 0 & 4 & 7 & 10 \\
\hline & & Urine & 0 & 4 & 7 & 10 \\
\hline & & Saliva & 0 & 4 & 7 & 10 \\
\hline & & Lesion T12 & 0 & 1 & 3 & 5 \\
\hline & \multirow{4}{*}{ TB culture } & Feces & 0 & 12 & 21 & 30 \\
\hline & & Urine & 0 & 12 & 21 & 30 \\
\hline & & Saliva & 0 & 12 & 21 & 30 \\
\hline & & Lesion T12 & 0 & 8 & 14 & 20 \\
\hline & $\mathrm{X}$-ray AP & Vert. 12 & 0 & 8 & 14 & 20 \\
\hline & X-ray Lat. & Vert. 12 & 0 & 8 & 14 & 20 \\
\hline & $\mathrm{X}$-ray AP & Lungs & 0 & 12 & 21 & 30 \\
\hline & X-ray Lat. & Lungs & 0 & 12 & 21 & 30 \\
\hline & Histopathology & Lesion T12 & 0 & 1 & 3 & 5 \\
\hline Total & & & & & & 285 \\
\hline
\end{tabular}

TABLE 3: T12 lesion examination.

\begin{tabular}{|c|c|c|c|c|c|c|c|c|c|c|c|c|c|c|c|}
\hline \multirow{2}{*}{ Rabbits } & \multicolumn{3}{|c|}{$\mathrm{W}-1$} & \multicolumn{4}{|c|}{ W-6 } & \multicolumn{4}{|c|}{ W-10 } & \multicolumn{4}{|c|}{ W-14 } \\
\hline & PCR & AFB & Culture & PCR & AFB & Culture & HP & PCR & AFB & Culture & HP & PCR & AFB & Culture & HP \\
\hline G3 & Pos. 3 & Neg. & Neg. & & & & & & & & & & & & \\
\hline G4 & & & & Neg. & Neg. & Neg. & Pos. 2 & & & & & & & & \\
\hline G5 & & & & & & & & Neg. & Neg. & Neg. & Neg. & & & & \\
\hline G6 & & & & & & & & & & & & Neg. & Neg. & Neg. & Neg. \\
\hline
\end{tabular}

Radiological imaging of thoracolumbar anteroposterior and lateral projection for rabbits groups G3, G4, G5, and G6 showed also negative results.

Potential spread score of rabbit 3 and rabbit 4 is $1.75 \%$ ( 0 to $9 \%$ ) and $1.05 \%$ (0 to $9 \%)$. Infected score of rabbit 1 and rabbit 2 was $0 \%$.

\section{Discussion}

We conducted evaluations through examinations of PCR, $\mathrm{AFB}$, and culture on feces, urine, and saliva samples of control and treatment rabbits, histopathological examination of lesion tissue thoracal 12 of treatment rabbits, and radiological examination after the incubation period to treatment rabbits.

We developed two methods of potential spread assessment, which are assessment based on examinations of potential spread on rabbits of treatment group which we call "potential spread scoring" (Tables 1 and 4) and assessment based on examinations on rabbits of control group which we call "infected scoring system."

The examination results of PCR, AFB, and culture on feces, urine, and saliva samples of control and treatment rabbits which were taken in weeks $1,6,10$, and 14 were negative
TABLE 4: Potential spread score.

\begin{tabular}{lcc}
\hline & Potential spread score & Meaning \\
\hline Rabbit 3 & $1.75 \%$ & Not potential \\
Rabbit 4 & $1.05 \%$ & Not potential \\
Rabbit 5 & 0 & Not potential \\
Rabbit 6 & 0 & Not potential \\
\hline
\end{tabular}

Score from 0 to 9 is not potential; score from 10 to 39 is low potential; score from 40 to 69 is medium potential; score from 70 to 89 is high potential; score from 90 to 100 is very high potential.

$(-)$, as well as the examination results of AFB and culture on samples of lesions tissue of thoracal 12. On the other hand, the examination result of PCR was positive $3(+3)$ on liquid lesions sample of thoracal 12 from the treatment rabbit of incubation in week 1 (G3). The result of histopathological examination was positive $2(+2)$ on lesions tissue samples of thoracal 12 from the treatment rabbit of incubation in week 6 (G4), whereas the results of histopathological examination of lesions tissue samples of thoracal 12 from treatment rabbits of G5 and G6 were negative (-).

The scoring results (Tables 2 and 5) showed that both rabbits of control group (G1 and G2) were not potential to be infected because the potential spread scoring was of $0.00 \%$, 


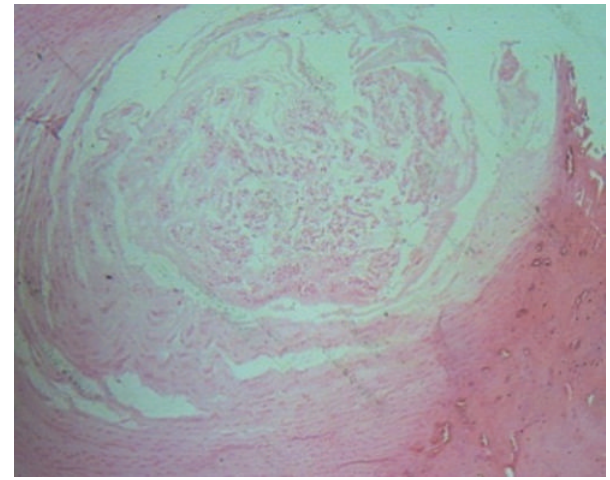

FIgURE 1: Histopathology examination of T12 lesion on Rabbit G3. There were no changes seen in the bone marrow, but there were inflammatory cells, fibrosis tissue, and capsule image seen corresponding to bacterial infection by Mycobacterium tuberculosis. There were also necrotic tissue and cell formation resembling Datia Langhans cell (zoom in the picture). Caseous process was not seen clearly but it was incomplete. The process of bone remodeling was clearly seen.

TABLE 5: Infected score.

\begin{tabular}{lcc}
\hline & Infected score & Meaning \\
\hline Rabbit 1 & 0 & Not infected \\
Rabbit 2 & 0 & Not infected \\
\hline
\end{tabular}

while, from the rabbits of treatment group, potential spread scoring of treatment rabbit 3 (G3) was of $1.75 \%$ and treatment rabbit 4 (G4) was of $1.05 \%$. This means that although there were positive results obtained from the examination, there was no potential to spread to the environment.

These results indicated that using the inoculation method of Mycobacterium tuberculosis suspension of $10^{6} \mathrm{cfu} / \mathrm{mL}$ directly into the corpus and incubating the rabbit until week 14, there was not any potential spread of Mycobacterium tuberculosis to the environment. The environment assumed here was the area around the location of rabbits treatment/incubation, including living creatures contacting directly (animal care workers, veterinarians, operator doctors, and rabbit control) or indirectly (the place of feces and urine disposal) with treatment rabbits.

This could mean that the method used did not reveal the potential spread of Mycobacterium tuberculosis into the environment, or perhaps the rabbit body's immune reaction was able to eliminate the infection of Mycobacterium tuberculosis completely, especially in the first weeks of inoculation, where there was no direct or hematogenous spread on the area around infected corpus.

In the inoculation method, we used Mycobacterium tuberculosis of $10^{6} \mathrm{cfu} / \mathrm{mL}(0.1 \mathrm{~mL}$ suspension with a quantity of $10^{7} \mathrm{cfu} / \mathrm{mL}$ ). It was based on a study of Crémieux and Carbon [8] which had successfully created a skin tuberculosis model on New Zealand rabbits through a liquefaction method on skin. Bacteria used were Mycobacterium tuberculosis of H37RV strain from the collection of the Laboratory of Clinical Microbiology FKUI. This is a pure strain of bacteria so that the virulence was probably not strong enough to fight against the rabbits' immune system. The use of bacterial strains with stronger virulence of course can be considered in future studies.

The difference between the procedure of infection induction performed to rabbit models of spondylitis tuberculosis and the procedure performed to rabbit models of tuberculosis infection in the lungs is on the bacterial inoculation technique. In the creation of rabbit models of tuberculosis in the lungs, a closed aerosol pump system was used in which the suspension or liquefaction of Mycobacterium tuberculosis was made into the form of droplet nuclei and was inoculated using inhalation directly into the lungs. This caused the whole treatment procedure to be performed at the facilities of ABSL3, considering that the natural spread of Mycobacterium tuberculosis is through droplet nuclei entering the lungs. On the other hand, the creation of rabbit models of spondylitis tuberculosis, to date there has not been supporting literature found so that we performed the approach through the method done by Converse et al. [4], Poelstra et al. [9], Schulz et al. [10], Bierry et al. [6], and several other researchers who had successfully created animal models with bone infection (osteomyelitis) using the technique of direct inoculation/injection into the bone.

We drilled the central part of the corpus thoracal 12 of $6-$ $10 \mathrm{~mm}$ depth; injected bacterial suspension of Mycobacterium tuberculosis into the hole formed and immediately covered them with subcutis fat. Mycobacterium tuberculosis is facultative aerobic bacteria, so the procedure was likely to cause the limitation of area and the availability of oxygen that decreased the ability of bacteria to survive and to breed. By extending the area of inoculation, it is expected that Mycobacterium tuberculosis will have enough space and oxygen to survive and to proliferate.

Histopathological examination was performed on corpus tissue of thoracal 12 obtained from surgical procedures. From macroscopic observation, bluish-colored tissue was found in the center of the corpus, which was the former location of drilling and injection of bacterial suspension of Mycobacterium tuberculosis. The formation of bluish tissue which was predicted as the area of the infection was probably due to the effort of rabbit's body in achieving bone healing process or due to the immune process of rabbits in striving to eliminate bacterial infection in the area. On the other hand, this could also be a manifestation of the proliferation process of the bacteria to survive. In histopathological examination procedure, we chose this tissue to do the cutting and the creation of tissue staining slides.

The results of staining and microscopic observation on tissue staining slides showed an image of spheres containing a lot of inflammatory cells, fibrosis tissue, and capsule image corresponding to bacterial infection of Mycobacterium tuberculosis $[8,9]$. There were also necrotic tissue and cells formation resembling Datia Langhans cells which were not clearly seen and an image of incomplete caseous process, whereas the process of bone remodeling was clearly seen. From these macroscopic and microscopic images, we concluded that there has been a process of bacterial infection of 


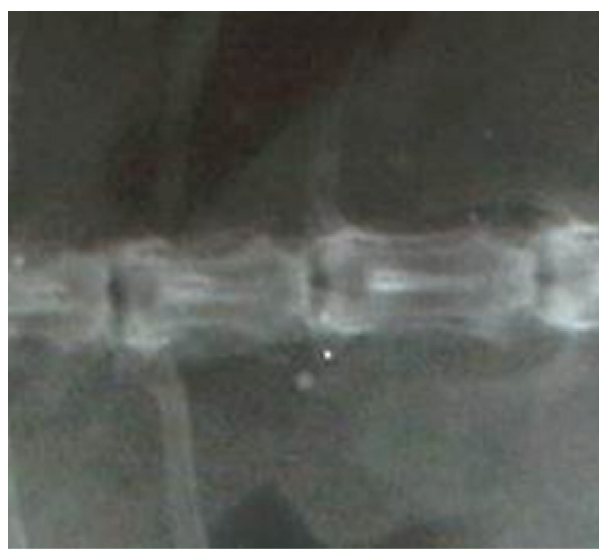

(a)

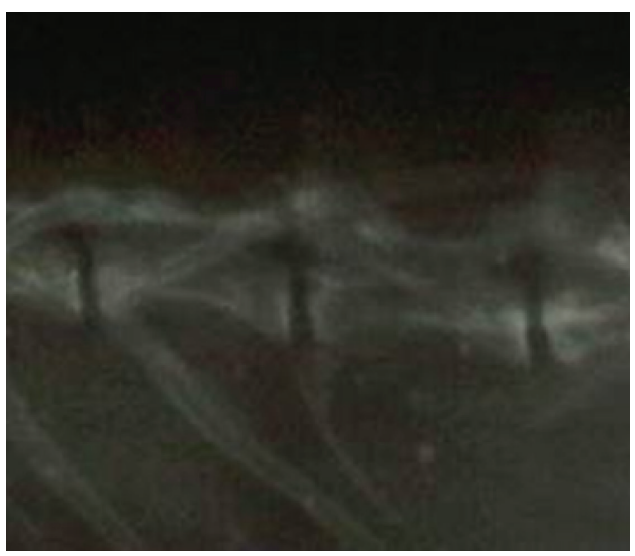

(b)

FIGURE 2: Plain X-ray image of T12 rabbit G6, 14 weeks after treatment. (a) Antero posterior projection: there is no clear vertebral destruction, but there is paravertebral shadow that indicates the abscess mass. (b) Lateral projection: there is slight destruction of T12 vertebral body that indicates the process infection.

Mycobacterium tuberculosis in the lesions of corpus thoracal 12.

Radiological observations showed that there was no clear destruction image as a result of the infection process. This was probably because Mycobacterium tuberculosis has a slow proliferation character so that the process of infection appeared in week 14 as early stages of chronic infection. In general, the destruction image from the X-ray imaging will be clearly seen when the destruction was large and was a prolonged process. Pus image was not seen which was probably because tissue reaction to the infection process had not yet appeared significantly.

Using the procedure of direct inoculation of $0.1 \mathrm{~mL}$ of Mycobacterium tuberculosis $10^{7} \mathrm{cfu} / \mathrm{mL}$ in the corpus of thoracal 12 with incubation period of 14 weeks, there was no potential spread of bacteria into the environment on spondylitis tuberculosis rabbit models (potential spread score $<9)$ and there is no spread on the control rabbits (infected score $<9)$ as shown on Table 1.

\section{Conflict of Interests}

The authors have no financial conflict of interests.

\section{Acknowledgment}

The research has grant from Fakultas Kedokteran Universitas Indonesia, 2012.

\section{References}

[1] D. Schlossberg, Tuberculosis and Non Tuberculous Mycobacterial Infections, McGraw-Hill, 5th edition, 2006.

[2] S. Sapardan, Total Treatment of Tuberculosis of The Spine. A Rational Problem Solving Approach, Perpustakaan Universitas Indonesia, 2004.

[3] Y. C. Manabe, A. M. Dannenberg Jr., S. K. Tyagi et al., "Different strains of Mycobacterium tuberculosis cause various spectrums of disease in the rabbit model of tuberculosis," Infection and Immunity, vol. 71, no. 10, pp. 6004-6011, 2003.

[4] P. J. Converse, A. M. Dannenberg Jr., J. E. Estep et al., "Cavitary tuberculosis produced in rabbits by aerosolized virulent tubercle bacilli," Infection and Immunity, vol. 64, no. 11, pp. 47764787, 1996

[5] L. Tsenova, R. Harbacheuski, E. Ellison, C. Manca, and G. Kaplan, "Aerosol exposure system for rabbits: application to Mycobacterium tuberculosis infection," Applied Biosafety, vol. 11, no. 1, pp. 7-14, 2006.

[6] G. Bierry, F. Jehl, G. Prévost et al., "Percutaneous inoculated rabbit model of intervertebral disc space infection: magnetic resonance imaging features with pathological correlation," Joint Bone Spine, vol. 75, no. 4, pp. 465-470, 2008.

[7] G. Zhang, B. Zhu, W. Shi, M. Wang, Z. Da, and Y. Zhang, "Evaluation of Mycobacterial virulence using rabbit skin liquefaction model," Virulence, vol. 1, no. 3, pp. 156-163, 2010.

[8] A.-C. Crémieux and C. Carbon, "Experimental models of bone and prosthetic joint infections," Clinical Infectious Diseases, vol. 25, no. 6, pp. 1295-1302, 1997.

[9] K. A. Poelstra, N. A. Barekzi, D. W. Grainger, A. G. Gristina, and T. C. Schuler, "A novel spinal implant infection model in rabbits," Spine, vol. 25, no. 4, pp. 406-410, 2000.

[10] S. Schulz, H. Steinhart, and R. Mutters, "Chronic osteomyelitis in a new rabbit model," Journal of Investigative Surgery, vol. 14, no. 2, pp. 121-131, 2001. 

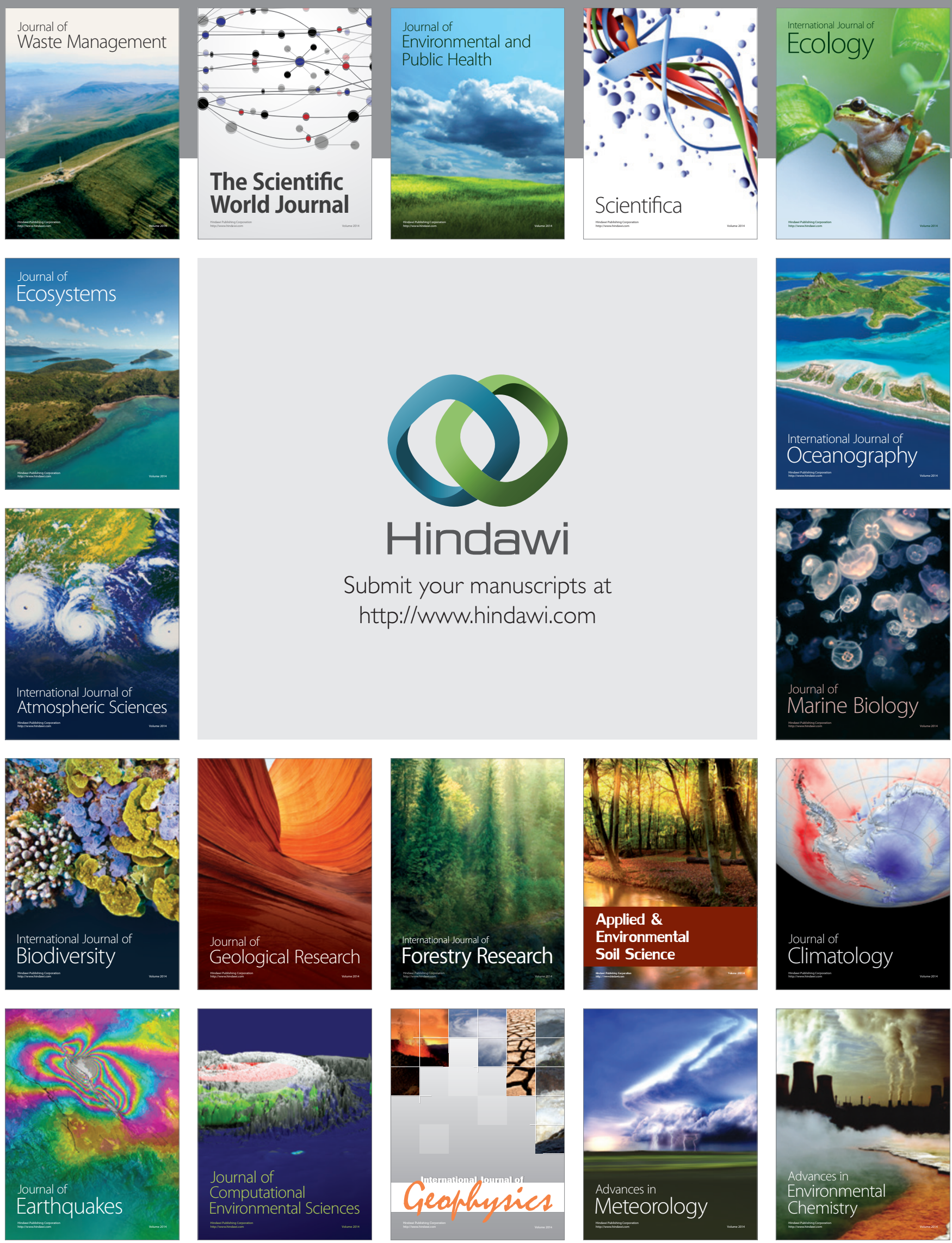